\title{
Das Schweizer Dorf der Gegenwart in geographisch-methodischer Sicht
}

Vielleicht sollte man in Nordamerika gereist sein, um unsere Dörfer neu schätzen zu können. Vielleicht muß man sich in den Landwirtschaftsgebieten der USA, in Kleinstädten und auf Farmen umgesehen haben, um das Fehlen des Dorfes bewußt zu erleben. Keine Dörfer, nur Stadt oder Einzelhof? Und man kehrt in die Schweiz zurück, in die dicht bevölkerte, traditionelle Kulturlandschaft, und entdeckt das Dorf neu, verstädtert zwar, mit vielen Ansätzen zur formalen Verflachung, aber gleichwohl Dörfer, überschaubar und doch gegliederte Lebensräume, profiliert durch eine unverwechselbare Geschichte, verortet in einmaliger naturräumlicher Position und ausgesetzt dem Kräftespiel der zivilisatorischen Entwicklung.

Ohne eine eingehende Diskussion des "Dorfi-Begriffes anzustreben, halten wir fest, daß der Terminus seinen sprachlichen Platz zwischen Weiler und Stadt innehat und daß mit der Vorstellung "Dorf» eine mäßig differenzierte Bevölkerung zu verbinden ist. Von der Siedlungsgeographie her mag die Definition von G. SCHWARZ eine erste quantitative Klarheit schaffen: «Die Gruppensiedlungen mittlerer Größe werden bei hinreichend permanenter Siedlungsart als Dörfer bezeichnet. Ihre untere Grenze hinsichtlich der Größe liegt etwa bei 20 und ihre obere mag durch rund 250 Wohnstätten gegeben sein» $(1966$, S. 111 f.). Als qualitative Ergänzung zu dieser Definition wäre noch die Bevölkerungs-, Siedlungs- und Erwerbsstruktur eines Dorfes zu kennzeichnen, etwa im Sinne der Agrarsoziologie vON BLANKENBURGs: "Als Dorf ist die baulich zusammenhängende Siedlung auf dem Lande anzusehen, deren Bevölkerung zu einem großen Teil existenzmäßig an den Boden gebunden ist, und zwar zunächst durch die beruflich auf die Landwirtschaft ausgerichtete Erwerbsbevölkerung, darüber hinaus aber auch dadurch, daß ein wesentlicher Teil der übrigen Bevölkerung eine Kleinsiedlung oder einen Garten besitzt. Die landwirtschaftliche Bevölkerung ist in der Einwohnerschaft des Dorfes relativ stark vertreten. Verglichen mit der Industrie- und Stadtsiedlung ist die Einwohnerzahl des Dorfès gering. Das Dorf stellt sich daher auch leicht als geschlossenes und überschaubares soziales Gebilde dar und kann sich als solches gut im Bewußtsein der Einwohner realisieren» (1962, S. 152).

Zusammenfassend ließe sich das Dorf als ein räumlicher Typus definieren, der als eine geschlossene, dichter gebaute Gruppensiedlung des ländlichen Rau- mes in Erscheinung tritt, und dessen relativ einheitlich gefügte Bevölkerung durch Landbesitz, Lebensart und Erwerbstätigkeit stärker an den Boden gebunden ist.

Bevor wir die dörfliche Thematik, wie sie in dieser Geographica-Nummer zum Ausdruck kommt, kurz würdigen, sei einleitend des Weges gedacht, den die Schweizer Dorfforschung bis heute zurückgelegt hat. Einen wichtigen Markstein auf diesem Entwicklungsgang bildete das Jahr 1941, als mitten in der Bedrohung des letzten Weltkrieges der HANS J. WEHRLI gewidmete Sammelband «Das Schweizer Dorf» erscheinen konnte. Das Werk, durch ERNST WINKLER mit Unterstützung von HANS HOFER in überzeugender Dichte und Vielfalt konzipiert und heraugegeben, vermittelt einen lebendigen Einblick in die damalige Situation der Schweizer Dorfforschung. Sucht man darin nach den vorherrschenden Fragestellungen, so findet man zum einen das durch die Zürcher Schule von Prof. WEHRLI gepflegte entwicklungsgeschichtliche Prinzip vertreten. Davon zeugen klassisch gewordene Regionalstudien, wie sie auch an andern Universitätsinstituten der Schweiz ausgearbeitet worden sind. Eine weitere Forschungsaktivität hatte die Volkskunde in die geographische Dorfarbeit übertragen, indem spezifische Fragen des dörflichen Volkslebens auf räumlich faßbare Tatbestände angewandt wurden (vgl. etwa HUNZIKERs Studien über das Schweizerhaus). Was später als Landschaftsökologie die Umweltdiskussion beleben wird, beschäftigte schon 1941 die biologisch engagierten Geographen, so etwa EMIL EGLI in seinem Aufsatz über die Naturgrundlagen des Schweizer Dorfes. Des weiteren ergab sich aus der Zeitsituation heraus eine nationalpolitische Dorfproblematik, die sich in den Beiträgen von HANS BOESCH, ERNST LEEMANN u. a. kundtat. Nach E. WINKLER sollte der Geograph beantworten «ob das Dorf des schweizerischen Staates sich von den übrigen unterscheidet und wenn dies der Fall sein sollte, worin dann sein Sonderwesen begründet ist. Diese Aufgabe zu lösen ist naturgemäß nur durch umfassende Vergleiche mit den Dorftypen und -Individualitäten des Auslandes möglich» (1941, S. 22). Wurden auch viele Dorfstudien von Nachbardisziplinen geleistet, so blieb - nach WINKLER (1941) -

Prof. Dr. W. A. Gallusser, Geogr. Institut der Universität Basel, Klingelbergstraße 16, 4056 Basel 
die geographische Hauptfrage nach dem ganzheitlichen und dem dynamischen Wesen des Dorfes wissenschaftlich offen: «Eine auf das wirkliche Sein der konkreten Welt gerichtete und damit Vergangenheit, Gegenwart und Zukunft, Zustand und Vorgang, Materie und Geist, Form und Inhalt gleichermaßen bewertende Forschung dagegen muß das Dorf sowohl als statistisches wie dynamisches Objekt und ferner nach seinen sämtlichen räumlichen und zeitlichen Bindungen interessieren und zur geistigen Erfassung auffordern» (a.a.O., S. 21).

Seitdem hat sich nun die Entwicklung der Dorfforschung reich differenziert und eine Zusammenfassung der wissenschaftlichen Ergebnisse wäre verdienstvoll; für die Zeit um 1950 bestehen zwar einige geographische Übersichten, so etwa die gemeindeanalytische Einführung in die "Landschaften der Schweiz» von H. GUTERSOHN (1950) und das dem Schweizer Dorf gewidmete Heimatbuch von w. U. GUYAN (1948). Aus Platzgründen muß uns eine ausführlichere Würdigung der neueren Arbeiten über das Schweizer Dorf versagt bleiben. Die vorliegenden Dorfstudien mögen aber im Sinne einer Zwischenbilanz einige Trends dieser Entwicklung aufzeigen.

\section{$*$}

Der Beitrag über die Klettgau-Gemeinde Osterfingen vertritt in unserem Sonderheft eine traditionelle Forschungsrichtung, nämlich die durch Kollegen GUYAN seit Jahrzehnten vertiefte Methodik der Landschaftsgeschichte. Dieses bewährte Arbeitsverfahren gewinnt heute durch sein Aussagevermögen für die Ortsbildund die Landschaftsschutz-Planung eine zusätzliche Bedeutung.

Die übrigen Artikel entstanden alle im Rahmen des Forschungsprogrammes "Kulturlandschaftswandel in der Schweiz», worüber in "Geographica Helvetica»1974, Nr. 2/3 ausführlich berichtet worden ist. Wesentliches Kennzeichen des KLW-Programmes ist die Vergleichbarkeit von gemeindlichen Struktur- und Entwicklungsmerkmalen ausgewählter Testgemeinden. Die Durchführung der Arbeit obliegt einer eigentlichen Forschungsgemeinschaft von Geographen aus allen Landesgegenden und von Vertretern aller geographischen Hochschulinstitute der Schweiz, wodurch trotz der Einheitlichkeit in der Zielsetzung und im Grundsätzlichen eine fachlich und regional differenzierte Methodik möglich und notwendig wird. Diese Vielfalt von einzelnen Teilstudien widerspiegelt einerseits den Reichtum an Fragestellungen innerhalb des KLWProgrammes, andererseits die dem räumlichen Objekt «Testgemeinde» innewohnende Vielschichtigkeit. Jedenfalls erhält die heutige Dorfforschung durch die Impulse der KLW-Studien eine zusätzliche Förderung, indem - wegen des regionalen Auswahlprinzips - die wichtigsten Dorftypen unseres Landes einer gründlichen Struktur- und Entwicklungsanalyse unterzogen werden. $\mathrm{Da}$ dabei die ausgreifende historisch-landeskundliche Untersuchung nicht zum Zuge kommt, ist methodischer Zwang und nicht etwa eine Minderbewertung der langfristig orientierten historischen Geographie. Der aktualgeographische Aspekt bleibt trotz seiner zeitlichen Einschränkung methodisch reichhaltig und entwicklungsfähig, wie dies aus den übrigen Beiträgen dieser Nummer deutlich wird, denn trotz des gemeinsamen Forschungsetiketts «KLW» hatten alle Autoren freie Hand bei der Darstellung ihrer Testgemeinden.

K. AERNI und R. TUOR befassen sich mit Gelterfingen als einer landwirtschaftlichen Testgemeinde im Berner Mittelland. Ihre Analyse betrifft gesamthaft die wesentlichsten Elemente des Kulturraumes, erstrebt demnach ein strukturelles Verständnis für den dörflichen Lebensraum und betont zudem - über den engeren KLW-Ansatz hinaus - die historische Dimension der einzelnen Raumteile. Dabei ist die Berücksichtigung der Grundbesitzverhältnisse besonders geeignet, den örtlichen Zusammenhang zwischen Dorfsiedlung und Flur zu verdeutlichen. In dieser Bemühung um eine vertiefte kulturräumliche Strukturkenntnis und deren typisierende Zusammenfassung hat sich denn auch die Berner Geographenschule verdient gemacht: die Karte über «Bäuerliche Siedlungs- und Flurformen» der Schweiz (1973) und die «Raumtypisierung nach geographischen Gesichtspunkten» (1974) von GEORGES GROSJEAN dürfen als geographische Landmarken für die Schweizer Dorfforschung bezeichnet werden.

In seinem Aufsatz über Breno im Malcantone versucht W. LEIMGRUBER, die dörfliche Problematik vor allem bevölkerungsanalytisch klarzulegen, ausgehend von der unausgesprochenen Annahme des Menschen als "agent modificateur" des Raumes. Die eindringliche Kenntnis der bevölkerungs- und sozialgeographischen Sachverhalte des Dorfes sollten in der Schweizer Geographie noch vermehrt angestrebt werden, wie man dies z. B. in einer Untersuchung von zehn ausgewähl- 
ten Landgemeinden der Nordwestschweiz versucht hatte (GALLUSSER, 1970). Beim Aufsatz von F. CHIFFELLE und J. RYCHNER über La Sagne im Neuenburger Hochjura wird die dörfliche Demographie durch den Einbezug landwirtschaftlicher Aspekte bereichert, und zwar im Sinne einer ökonomisch orientierten «Géographie rurale», welche CHIFFELLE speziell für die Neuenburger Agrarlandschaft entwickelt hat (CHIFFELLE, 1968).

Entsprechend den Zielsetzungen der Zürcher Geographenschule von G. FURRER, behandelt $\mathrm{CH}$. STOCKER das Dorf Urmein am Heinzenberg im thematisch erweiterten Rahmen des alpinen Kulturlandschaftswandels: die Verfasserin versteht es, ausgewählte Strukturkennzeichen des Bergdorfes in ihrem Zusammenhang mit der übergeordneten Problematik einer alpinen Entwicklungsregion aufzuzeigen. Was eine aktualdynamisch ausgerichtete Dorfgeographie zu erreichen vermag, zeigt die Studie von L. BRIDEL über Bottens im Einflußbereich von Lausanne. Verständlicherweise ist der Gehalt der offiziellen KLW-Analyse von Bottens, wie sie 1975 publiziert worden ist, im vorliegenden Artikel nur ansatzweise zu vermitteln; doch klärt BRIDEL die gesamthafte Situation von Bottens methodisch überzeugend, indem er das Dorf explizit im Hinblick auf zusammenwirkende raumdynamische Prozesse (Veränderung von Bodennutzung und Lebensweise, Grundstücksmarkt, Regionalplanung) untersucht.

Unter dem Einfluß der allgemeinen Verstädterung ist das Schweizer Dorf als traditionsreicher Lebensraum zur Veränderung gezwungen worden. Diese Tatsache verleiht der Schweizer Dorfgeographie ein neues Gewicht, sofern sich deren Methodik als offen erweist gegenüber dem verpflichtenden Reichtum eines tradierten Formenschatzes und der verletzlichen Lebensgrundlage des natürlichen Milieus, sowie gegenüber der Herausforderung einer im Aufbruch befindlichen humanen Umwelt. In der fundierten Analyse wie in der synthetischen Rekonstruktion vermag die geographische Arbeit beizutragen zu einem neuen Verständnis der dörflichen Lebensformen, woraus entwicklungsfähige Alternativen zur gegenwärtigen Verstädterungsbewegung erwachsen könnten. In dieser Hinsicht leistet die Dorfgeographie von heute einen grundsätzlichen Beitrag zur Modellierung unserer Lebensräume von morgen.

\section{Literaturhinweise}

BLANKENBURG vON, P.: Einführung in die Agrarsoziologie. Stuttgart 1962. $170 \mathrm{~S}$.

BOESCH, H.: New Glarus (Wis.). Eine Schweizersiedlung in den Vereinigten Staaten. In «Das Schweizer Dorf», S. 286-299. Zürich 1941

BRIDEL, L.: Bottens 1975. Vervielfältigter Bericht des Geograph. Instituts der Universität Lausanne. Lausanne 1975. $156 \mathrm{~S}$.

CHIFFELLE, F.: Le Bas-Pays neuchâtelois. Etude de géographie rurale. Neuchâtel 1968. $351 \mathrm{~S}$.

EGLI, E.: Die Naturgrundlagen des Schweizer Dorfes. In «Das Schweizer Dorf», S. 23-40, Zürich 1941

GALLUSSER, W. A.: Struktur und Entwicklung ländlicher Räume der Nordwestschweiz. Aktualgeographische Analyse der Kulturlandschaft im Zeitraum 1955 bis 1968. Basler Beiträge zur Geographie 11, Basel 1970. $324 \mathrm{~S}$.

DERS. und BUCHMANN, w.: Der Kulturlandschaftswandel in der Schweiz als geographisches Forschungsprogramm. In «Geographica Helvetica» Nr. 2/3, S. 49 bis 70. Bern 1974

GROSJEAN, G.: Bäuerliche Siedlungs- und Flurformen, Übersicht. Karte Nr.38 (mit Text) im "Atlas der Schweiz». Bern 1973

DERS.: Raumtypisierung nach geographischen Gesichtspunkten als Grundlage der Raumplanung auf höherer Stufe. Geographica Bernensia, P1. Bern 1974. 197 S. (excl. Anhang)

GUTERSOHN, H.: Landschaften der Schweiz. Zürich 1950. $218 \mathrm{~S}$.

GUYAN, w. U.: Das Schweizer Dorf. Schweizer Heimatbücher 26. Bern 1948. $48 \mathrm{~S}$.

HUNZIKER, J.: Das Schweizerhaus nach seinen landschaftlichen Formen und seiner geschichtlichen Entwicklung. Aarau $1900 \mathrm{ff}$.

LEEMANN, E.: Das Schweizerdorf als Lebensgemeinschaft. In «Das Schweizer Dorf», S. 300-316. Zürich 1941 SCHWARZ, G.: Allgemeine Siedlungsgeographie. Berlin $1966,751 \mathrm{~S}$.

wINKLER, E. (Hg.): Das Schweizer Dorf. Beiträge zur Erkenntnis seines Wesens. (Mit Literaturverzeichnis.) Zürich 1941. $422 \mathrm{~S}$.

DERS.: Ziele und Wege schweizerischer Dorfforschung. In «Das Schweizer Dorf» S. 9-22. Zürich 1941 